\title{
A stabilized formulation with maximum entropy meshfree approximants for viscoplastic flow simulation in metal forming
}

\author{
F. Greco • L. Filice • C. Peco • M. Arroyo
}

Received: 15 October 2013 / Accepted: 7 March 2014

(C) Springer-Verlag France 2014

\begin{abstract}
The finite element method is the reference technique in the simulation of metal forming and provides excellent results with both Eulerian and Lagrangian implementations. The latter approach is more natural and direct but the large deformations involved in such processes require remeshing-rezoning algorithms that increase the computational times and reduce the quality of the results. Meshfree methods can better handle large deformations and have shown encouraging results. However, viscoplastic flows are nearly incompressible, which poses a challenge to meshfree methods. In this paper we propose a simple model of viscoplasticity, where both the pressure and velocity fields are discretized with maximum entropy approximants. The inf-sup condition is circumvented with a numerically consistent stabilized formulation that involves the gradient of the pressure. The performance of the method is studied in some benchmark problems including metal forming and orthogonal cutting.
\end{abstract}

Keywords Maximum entropy · Metal forming · Viscoplasticity $\cdot$ Stabilization

\section{F. Greco $(\bowtie) \cdot$ L. Filice}

Department of Mechanical, Energy and Management Engineering, University of Calabria, Rende 87036, Italy

e-mail: francesco.greco@unical.it

C. Peco $\cdot$ M. Arroyo

LaCàN, Universitat Politècnica de Catalunya (UPC), Barcelona 08034, Spain

\section{Introduction}

The finite element method (FEM) has been successfully applied to the simulation of all types of metal forming process $[1,2]$ and, thanks to the increasing computer power, it can provide excellent results with reasonable computational times. Thanks to its flexibility and robustness commercial FEM codes are a standard tool in the industry.

However, the main limitation of the FEM in this kind of application is that the quality of the results depends on the mesh [3]. If a Lagrangian formulation is used the mesh moves with the material and, due to the high distortions, the numerical results loose their accuracy, unless remeshingrezoning techniques are used. This step becomes very time consuming in 3D. Furthermore additional errors are introduced when the variables are mapped from the old mesh to the new one. Metal forming has been also studied with Eulerian [4-6] and ALE formulations [7-9] which involve drawbacks such as determining the geometry of the free surface of the flow in the former case and controlling the mesh motion in the latter. For these reasons, even if the traditional FEM provides very good results in many applications, alternative techniques based on modified FEM formulations or on meshfree approximation schemes [10] appear an interesting alternative in the simulation of metal forming processes. Recently it has been shown that the application of nodal integration techniques to the FEM makes the method insensitive to mesh distortion and alleviates volumetric locking problems in the study of incompressible materials [11-13]. Thanks to this features the nodal integrated FEM appears particularly suited for metal forming and machining problems; however its application is still in the process of development [14-16].

On the other hand also meshless methods are wellsuited for this type of problems since their accuracy is not 
hydrostatic pressure around the nodes. In [58] this formulation is extended to Stokes flow in two dimensions and three-dimensional incompressible elasticity and its stability is demonstrated through inf-sup numerical tests. High order max-ent schemes [59-62] would be another option in order to employ richer approximants for the velocities. However, such approximation schemes are not guaranteed to be LBBcompliant if coupled with constant or linear approximants for the pressure.

It is also worth to note that volumetric locking problems may be avoided with the nodal integration approach proposed in [49], where mixed formulations are not necessary. However spurious low energy modes due to the averaging of the strain arise in nodal integration schemes and therefore other sorts of stabilizations are required anyway [63].

Recently the application of consistent stabilization techniques that have been widely studied in the FEM literature [64-66] has been also extended to max-ent methods for the resolution of the Stokes equations [67]. These techniques allow us to use equal order approximations for velocity and pressure and performed well in fluid dynamics benchmark applications [67]. Due to the analogy with the Stokes equations, in this work we extend this stabilization approach also to the simulation of visco, modification of the technique $r$ incompressibility equation with

Drt for finite elements

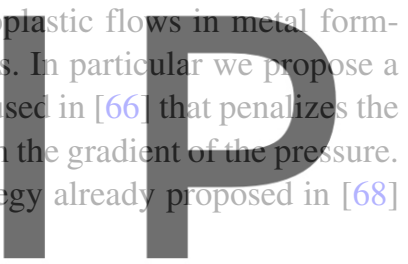

The outline of the paper is the following: firstly a ter for free at https//WWW.scipedia.com to

For fixed $\boldsymbol{x}$, minimize

$$
\sum_{a=1}^{N} \beta_{a} p_{a}\left|\boldsymbol{x}-\boldsymbol{x}_{a}\right|^{2}+\sum_{a=1}^{N} p_{a} \text { in } p_{a}
$$

subject to

$$
\begin{aligned}
& p_{a} \geq 0, \quad a=1, \ldots, N \\
& \sum_{a=1}^{N} p_{a}=1, \quad \sum_{a=1}^{N} p_{a} \boldsymbol{x}_{a}=\boldsymbol{x},
\end{aligned}
$$

\section{The local maximum entropy approximants \\ The max-ent basis functions, denoted by $p_{a}(\boldsymbol{x}), a=$ $1, \ldots, N$ with $\boldsymbol{x} \in R^{d}$, where $N$ is the total number of nodes and $d$ is the space dimension, are designed to be strictly non- negative and to fulfill the zeroth and first order consistency conditions

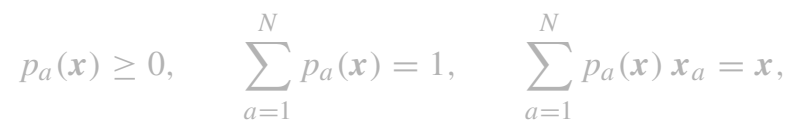 \\ where the last equation allows us to identify the vectorial weights $x_{a}$ with the positions of the nodes associated with each basis function. In this work we develop a meshfree method is extended to the present application, including some implementation aspects. Finally, in section "Numer- ical examples", some numerical examples on bench- mark metal forming and orthogonal cutting processes are shown.}

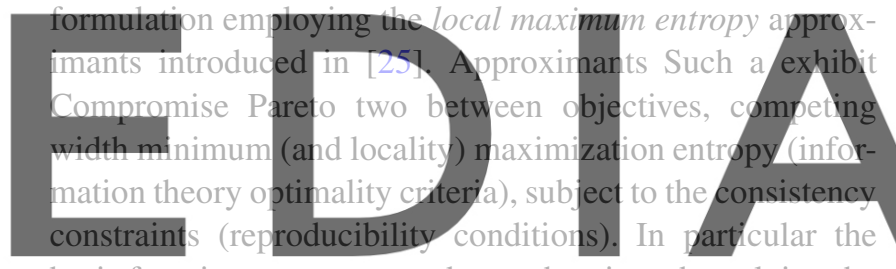

basis functions are computed at each point $x$ by solving the following constrained optimization problem

\section{lownload the version without the waterma}

where the set of non-negative nodal parameters $\left\{\beta_{a}=\right.$ $\left.\gamma_{a} / h_{a}^{2}\right\}_{a=1, \ldots, N}$ defines the locality of the approximants $[25,26]$. The dimensionless parameter $\gamma_{a}$ characterizes the degree of locality of the basis function associated to the node $\boldsymbol{x}_{a}$, while $h_{a}$ represents the nodal spacing. The basis functions become sharper and more local as the value of the dimensionless parameter $\gamma_{a}$ increases, and the Delaunay approximants arise as a specialized limit (for $\gamma_{a} \geq 4$ in practice), as illustrated in Fig. 1 for a one-dimensional domain.

As fully detailed in [25], it can be mathematically proved that the optimization problem has a unique solution. The efficient solution follows from standard duality methods.
Here, we summarize the recipe for the final calculation of the basis functions. By analogy with statistical mechanics, we define the partition function $Z(\boldsymbol{x}, \lambda)=\sum_{b=1}^{N} \exp \left[-\beta_{b}\left|\boldsymbol{x}-\boldsymbol{x}_{b}\right|^{2}+\lambda \cdot\left(\boldsymbol{x}-\boldsymbol{x}_{b}\right)\right]$ where the sum can be restricted to the nodes $\boldsymbol{x}_{b}$ in the vicinity of $\boldsymbol{x}$. At each evaluation point, the Lagrange multiplier for the linear consistency condition is the unique solution to a solvable, convex, unconstrained optimization problem

$\lambda^{*}(\boldsymbol{x})=\arg \min _{\lambda \in R^{d}} \ln Z(\boldsymbol{x}, \lambda)$. 
where $S_{f}$ is the flow stress and $d=\sqrt{\frac{2}{3} d_{i j} d_{i j}}$ is the equivalent strain rate. The viscosity then also depends on $d$ with the relation:

$\mu=\mu_{0}(\sqrt{3} d)^{n-1}$

where $C=3 \mu_{0}$.

The weak form of the equilibrium equation is:

$$
\begin{aligned}
\int_{\Omega} \nabla \boldsymbol{\sigma}: \delta \boldsymbol{d} d \Omega-\int_{\Omega} \boldsymbol{f} \cdot \delta \boldsymbol{v} d \Omega & =0 \\
\int_{\Omega} \nabla \cdot \boldsymbol{v} \delta p d \Omega & =0 .
\end{aligned}
$$

According to [65] the general way to stabilize the problem is to add to the aforementioned equation a consistent term in the form:

$$
\delta \Pi_{S}=\int_{\Omega} \tau \mathcal{P}(\delta v, \delta p) R(v, p) d \Omega
$$

where $R(v, p)$ is the residual of the strong form of the problem (which ensures the consistency of the new weak form), $\tau$ is a parameter which controls the amount of stabilization and $\mathcal{P}(\delta v, \delta p)$ is a partition of the differential operator. Choosing $\mathcal{P}(\delta v, \delta p)=\nabla \delta p$ we recover the simplified
Gquerkin Least Squares scheme that was firstly proposed
by Hughes [70] and it is also known as pressure-Polsson
stakilized Galerkin method [66]:
$\delta \delta_{S}=\int_{\Omega} \tau \nabla \delta p(\nabla \cdot \sigma-f) d S$

$=\int \tau \nabla \delta p(\nabla \cdot(2 \mu d)-\nabla p-f) d \Omega$ ter for $f_{\$ e}$ at https//www.scipedia.com to

As commonly done in finite element methods we neglect the second order term $\nabla \cdot(2 \mu d)$. Indeed, for low order finite elements, the first part of the consistent term is zero. A stabilization approach that involves only the gradient of the pressure has been also independently proposed in [68]. This simplification avoids the computation of the second derivatives of the basis functions, which can be cumbersome and ill-posed near the boundary $[28,69]$. Thus the stabilization is given only by:

$\delta \Pi_{S}=\int_{\Omega} \tau \nabla \delta p(-\nabla p-\boldsymbol{f}) d \Omega$

This formulation is still numerically consistent, by making the parameter $\tau$ dependent on the nodal spacing as proposed in [67] in the following way

$\tau=\frac{C_{s}}{\mu} \bar{\rho}^{2}$

For regular meshes $\bar{\rho}$ is equal to the nodal spacing $h$, while for irregular meshes excellent results were found calculating $\bar{\rho}$ as a simple average of the neighbours to a given integration point. In particular the following algorithm was used:

- calculate the Delaunay triangulation of the cloud of nodes; the triangulation is used also for the integration

- for each node calculate the local mesh size $h_{i}$ as a mean of the distance from its natural neighbours in the triangulation

- for each integration point calculate the max-ent shape functions using for the nodes $\beta_{i}=\gamma / h_{i}^{2}$

- for each integration point calculate $\bar{\rho}$ as a mean of $h_{i}$ of its neighbours

Note that $\tau \rightarrow 0$ with the mesh refinement and, therefore, the numerical method is consistent. This aspect is further discussed in $[66,71]$.

The choice of $C_{S}$ is in general problem dependent. For the finite elements this topic has been analyzed in [72] and is also discussed in [64]. In general, when $C_{S}$ is increased, the quality of the results in terms of regularity of the solutions is improved but the accuracy is worsened due to a higher error in the penalization. In our numerical experience we found that very good results are achieved with $C_{S}$ in the range from 0.5 to 2 and set $C_{s}=0.5$ in all the applications shown in

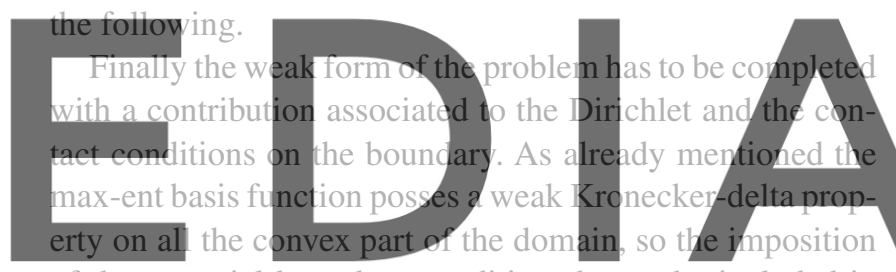
of the essential boundary conditions has to be included in the variational formulation only if it is required in some nondownload the version withqut the watermal approach the following term is then added to the functional to minimize:

$\Pi_{D}=\int_{\Gamma_{D}} \lambda_{D} \cdot\left(\boldsymbol{v}-\boldsymbol{v}_{\boldsymbol{d}}\right) d \Gamma$

As for contact [73], see to Fig. 4, for any point $\boldsymbol{x}_{s}$ on the work-piece (slave surface) a gap function is defined as the distance between $\boldsymbol{x}_{\boldsymbol{s}}$ and the closest point $\overline{\boldsymbol{x}}$ on the tool (master surface):

$g_{n}=\left(\boldsymbol{x}_{s}-\overline{\boldsymbol{x}}\right) \cdot \boldsymbol{n}$

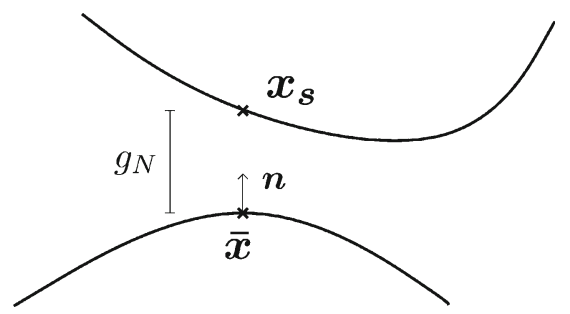

Fig. 4 The master and the slave surfaces and the gap function 
The new values of the viscosity give a new velocity field and the method is iterated until convergence. Although a slightly higher number of iterations is required, this approach is more robust than the Newton scheme. We noted that the best strategy is to combine the two schemes. The Direct Iteration Method is used to find an initial solution and then it is refined with the Newton scheme.

As mentioned above the numerical integration is performed using the Delaunay triangulation of the cloud of nodes in combination with the alpha-shape technique [75]. The Delaunay algorithm is the easiest way to obtain a triangulation for a given cloud of nodes and, respect to other meshing algorithms, its computational costs are negligible respect to the entire simulation [21]. The only problem of the Delaunay triangulation is that it is extended to the whole convex hull of the nodes. Therefore using the alpha-shape criterion the mesh has to be modified removing the triangles whose circum-radius is bigger than a given value. This approach has been already successfully applied in other reference works such as [46], where its application is discussed in detail. Once the mesh is obtained the integration is realized with standard Gauss quadrature methods within the triangles. Another possible approach to perform the numerical integration is to employ nodai integration schemes [12,
491. These schemes seem to avoid volunetric locking and
therefore do not need mixed formulations but the nodal
averaging of the strain leads to spurious low energy modes
yhich anyway requires stabilization techniques [63]. ter for free at https//www.scipedia.com to In this section the validity of the stabilized viscoplastic formulation that was proposed in the previous one is confirmed with some numerical results in the simulation of reference manufacturing processes. As already mentioned, the scope of this work is only to investigate on the possibilities of the new technique; therefore some simplifications are introduced in the modelling of the problems, such as neglecting friction and thermal phenomena. As far as the material behaviour is concerned the parameters for the Norton-Hoff power law are $\mu_{0}=150$ and $n=0.2$. According to [76] these values are typical of some aluminium alloys. In all the numerical applications the max-ent interpolants are constructed with $\gamma=1.6$.

\section{Upsetting}

A first benchmark example that was considered is the upsetting of a cylindrical billet that is progressively flattened between two plates. The domain represented in Fig. 5 is discretized with 524 nodes and 100 time steps $(\Delta t=1)$ are employed.
This process is a very good benchmark to test the efficiency of the stabilization because, due to the regularity of the domain, smooth pressure are expected and eventual oscillations can be easily detected. According to Fig. 5 the method performs very well and perfectly smooth maps are obtained for both the velocity and the pressure field.

A similar upsetting example has been studied in Reference [16] with the adaptive smoothed finite element method; in this application the accuracy of a standard FEM simulation is improved but spurious pressure oscillations are observed. Similar results would be obtained on using maxent or other meshfree methods with a non-stabilized nodal integration approach because of the nodal averaging of the strain.

\section{Extrusion}

Extrusion is a typical forming process, where the application of the finite element method is stressed by the heavy mesh distortions. For this reason and for the importance of its simulation in the industrial applications, this process has been widely studied in the literature on the meshless

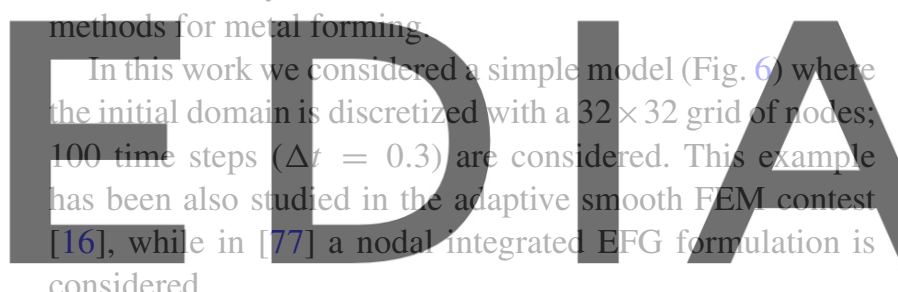

considered.

During the process the.shape of the profile is determined downhload the versign withoyt the waterma simplicity of this approach, also in this case very high quality maps are obtained for both the velocity and the pressure field, even in the zones where high deformations are present.

To assess the accuracy of the max-ent solution the process was also simulated with the commercial FEM code DEFORM $^{\mathrm{TM}}$, whose reliability in the simulation of forming processes is well recognised from more than 15 years [78]. In Fig. 7 a comparison is made between the prediction of the pressure trend with the two codes along the $x$ and the $y$ axes (see Fig. 6a) at the first step of the simulation. A good agreement between the curves can be observed on the symmetry axis $(x)$ and they are expected to match with mesh refinement. The two codes give the same prediction also along the $y$ axes in the first part of the bottom of the die and in the zone where the material is free to flow but the commercial code provides a higher prediction of the pressure peak. The smoothness of the max-ent basis functions precludes such a sharp trend with only a reduced number of nodes. However, the simplified model that has been employed in both simulations assumes a rigid and perfectly straight die (Fig. 6a) while in reality dies have some curvature and therefore the 
Fig. 5 Upsetting; (a) sketch of the geometry; (b-d) pressure at different time steps; (e) vertical velocity at the end of the process

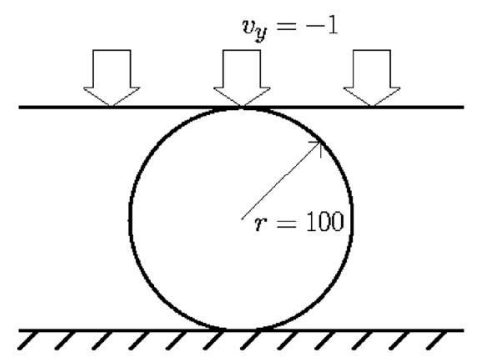

(a)

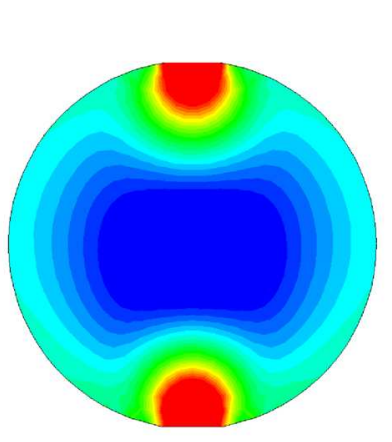

(b)

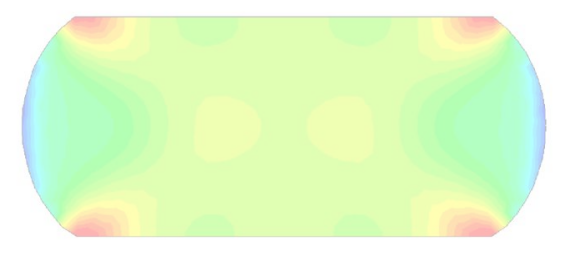

(c)

(d)
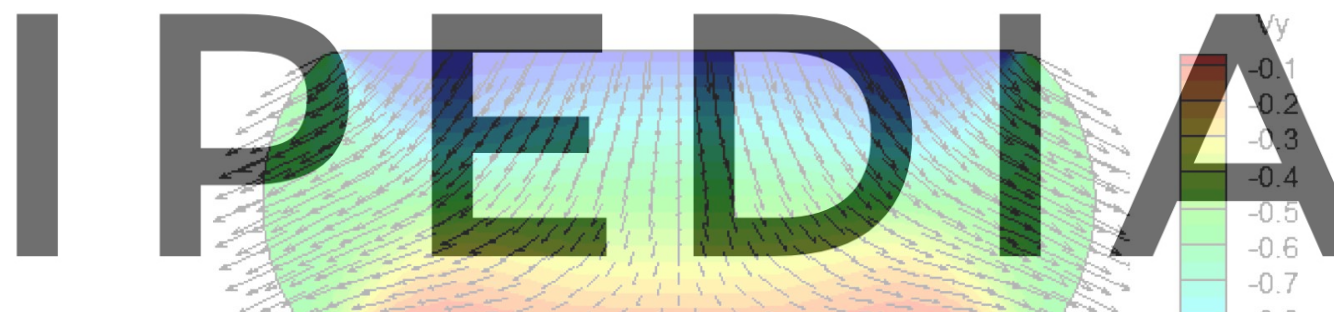

政

(e)

trend of the pressure field is more regular. This consideration is confirmed observing that, if the DEFORM ${ }^{\mathrm{TM}}$ die is smoothed with a curvature of a given radius $r$, the peak progressively decreases when $r$ is increased.

The reference solution of the DEFORM ${ }^{\mathrm{TM}}$ simulation was obtained with a very refined mesh; at the same time another simulation with a 1000 nodes mesh was considered in order to have an indicative comparison of the computational times. In particular, running the simulations on a standard $\mathrm{PC}$, the computational times were about an order of magnitude smaller for DEFORM ${ }^{\mathrm{TM}}$ as compared to the home-made max-ent code. This difference is not excessively high considering that the home-made code was implemented in the MATLAB ${ }^{\circledR}$ environment without any proper optimization effort. Probably more interesting is in fact to compare the computational costs of the two methods from a theoretical point of view. Max-ent basis functions are much more rapid to be calculated if compared to other meshless methods. According to section "The local maximum entropy approximants" their evaluation requires the subsequent resolution of a linear system given by the Newton-Raphson iterations; the resolution of these systems takes roughly the same time of the computation of the triangular FE shape functions in a given point [25]. The number of iterations is normally in the range of 4-8 for the interior points and increases up to 10 in the points close to the boundary of the domain. Another aspect to be taken into account is that the final max-ent stiffness matrix (as for other meshless methods) is denser than a standard FEM stiffness matrix due to the higher support of the shape functions, which also increases the computational times. According to Fig. 1 the extension of the basis functions can be regulated with the parameter $\gamma$. Higher values of $\gamma$ give more compact supports and are expected to reduce the 
Fig. 6 Extrusion; (a) sketch of the geometry; (b-d) pressure at different time steps; (e) horizontal velocity at the tenth step; (f) vertical velocity at the tenth step

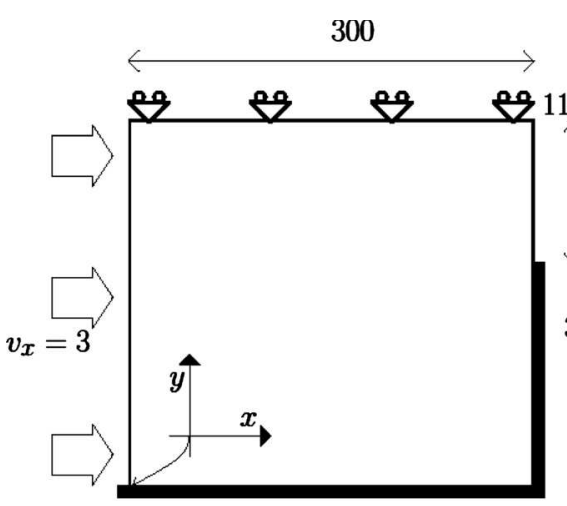

(a)

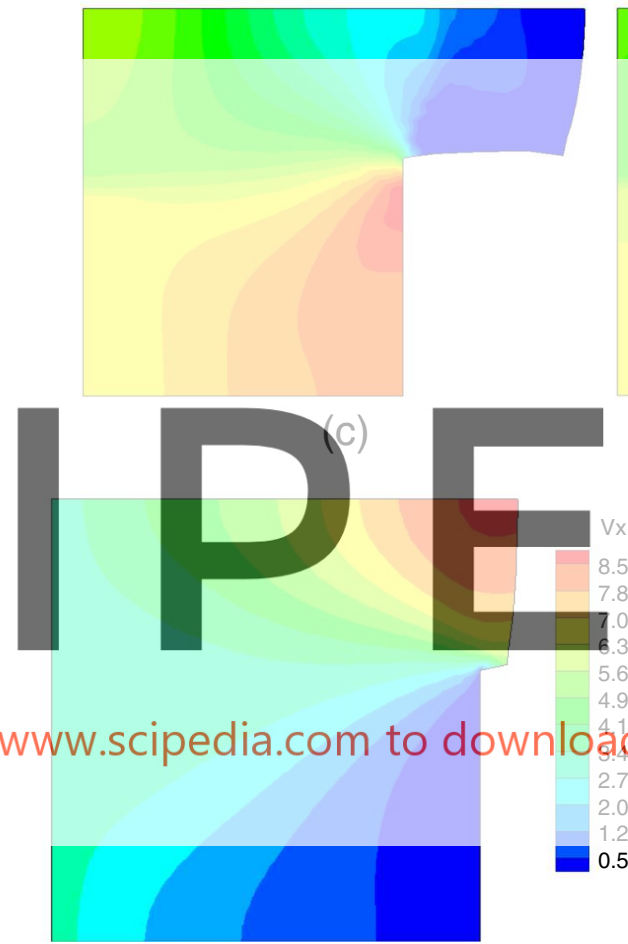

(e)

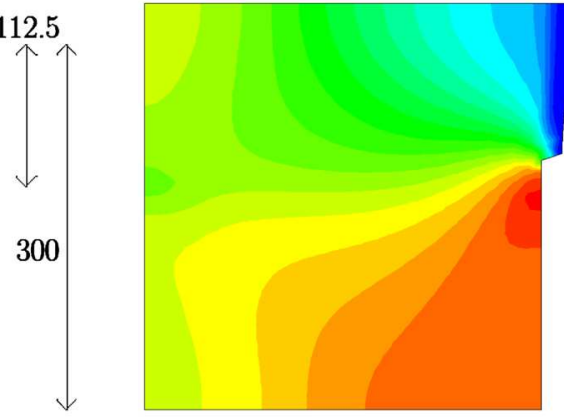

(b)
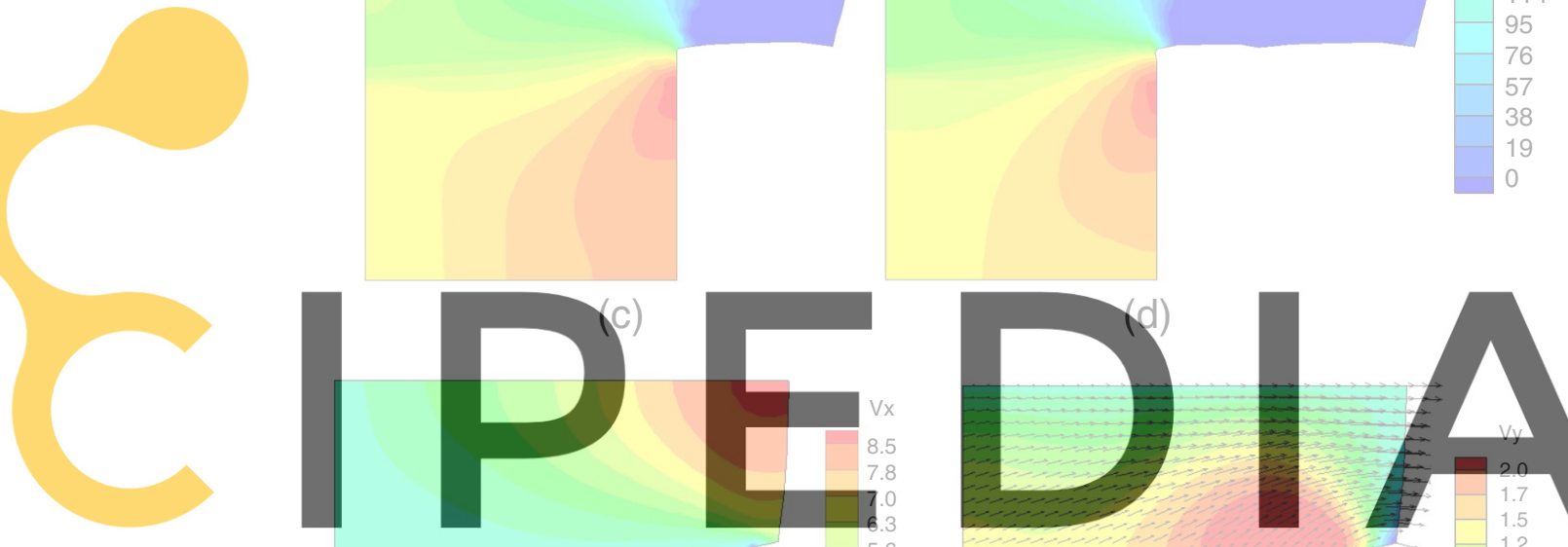

ter for free at https//www.scipedia.com to downlo âd the version without the waterimar

Fig. 7 Pressure along the symmetry axis $x$ for $y=300$ (left) and on the bottom of the die along the $y$ axis for $x=300$ (right) at the beginning of the simulation. A comparison between the max-ent and the DEFORM $^{\mathrm{TM}}$ predictions

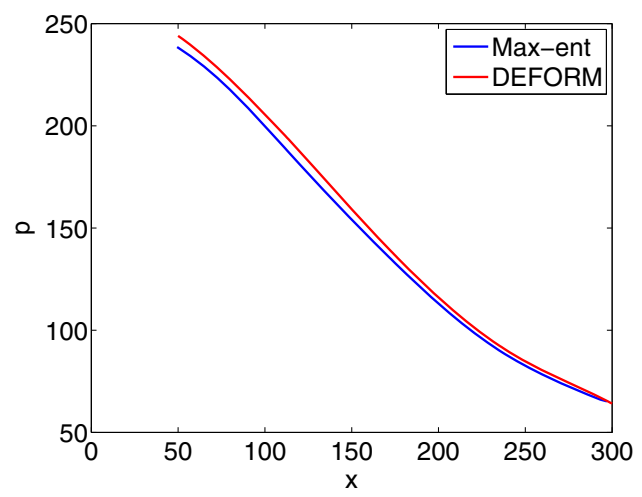

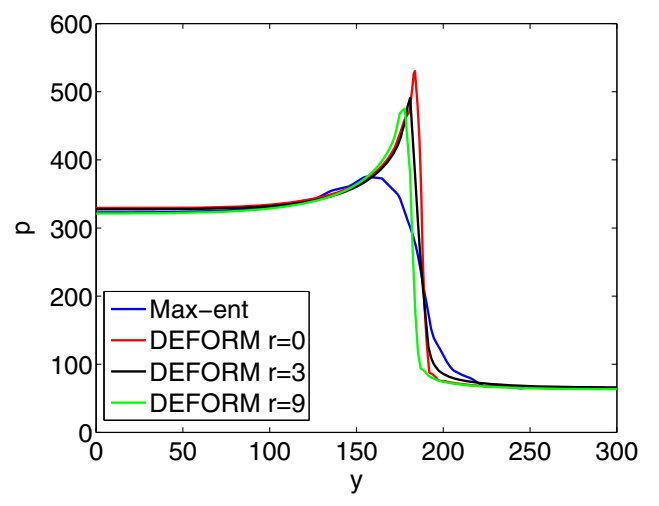


Fig. 8 Orthogonal cutting; (a) sketch of the geometry; (b-f) pressure at different time steps; (g) mesh at the $350^{\text {th }}$ step; (h) vertical velocity at the $350^{\text {th }}$ step

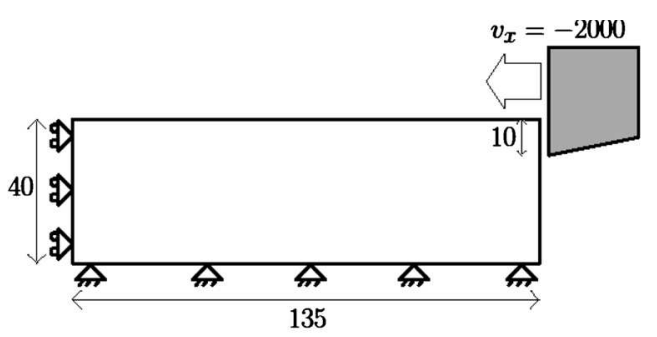

(a)

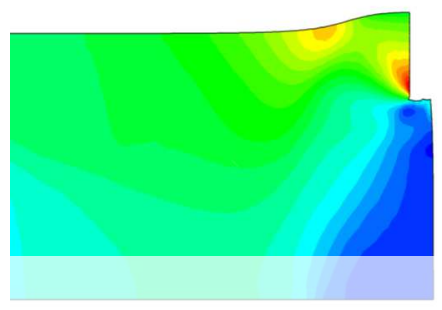

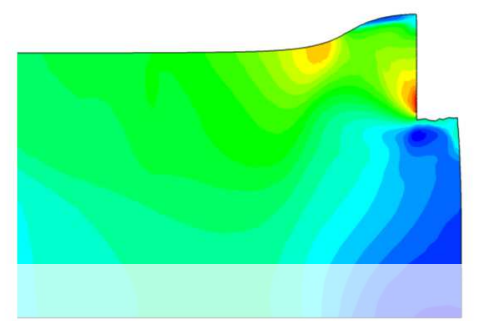

(b)

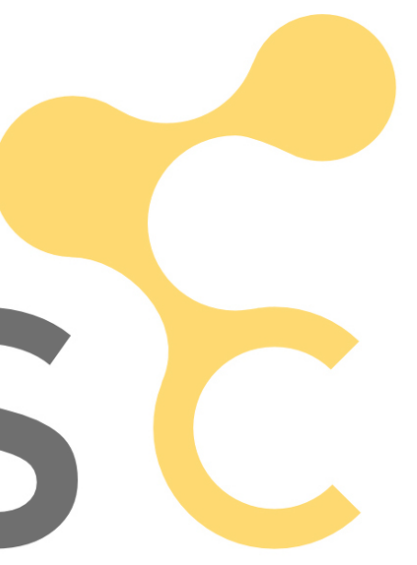

(c)

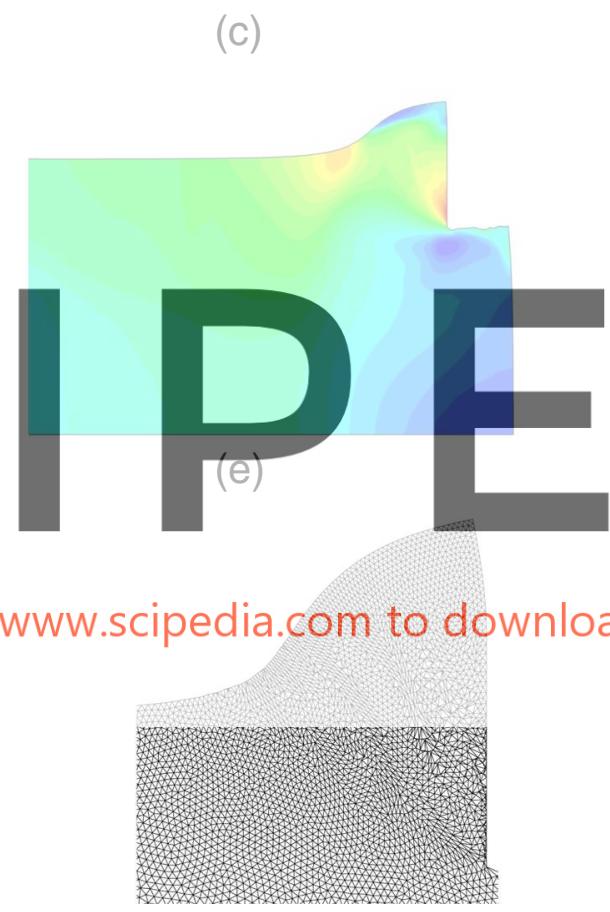

(g) (d)

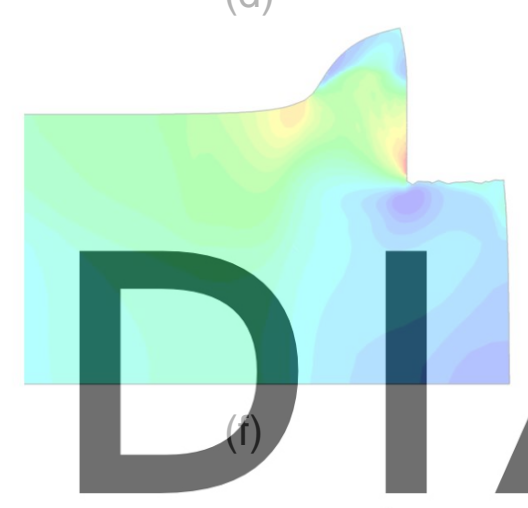

ter for free at https//www.scipedia.com to download the version without the vy

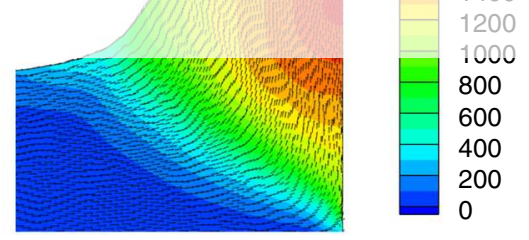

(h) computational times; on the contrary reducing $\gamma$ the accuracy can be improved at a higher computational cost. The value chosen in these examples $(\gamma=1.6)$ seems to be a good compromise.

\section{Orthogonal cutting}

The simulation of machining processes is another application where the mesh is heavily distorted during the analysis; therefore meshfree approximants may be a valid alternative to FEM. Here we consider a simple model of orthogonal cutting (Fig. 8). The domain is discretized using a 5000 nodes mesh with a node spacing $h \approx 0.33$ in the cutting zone. The simulation is subdivided in 350 time steps with an increment $\Delta t=2 \cdot 10^{-5}$. As we can see in Fig. 8b-f also in this application a regular trend for the pressure is obtained and the method performs well in the prediction of the pressure peak close to the tool tip. The results however degrade at the end of the simulation (Fig. 8f) due to uneven node distribution produced by the flow. As shown Fig. $8 \mathrm{~g}$ the mesh that was originally regular becomes heavily distorted and, in addition, the density of the nodes is no longer regular in some zones. Although the method performs well for such a distorted mesh (this would not be the case of 
finite elements) a rezoning would be beneficial to continue the simulation. This operation is not significantly time consuming when meshfree approximants are employed. In fact only the position of the nodes has to be changed and a new Delaunay mesh can be generated. This is not possible for high order finite elements that require more complicated and time consuming meshing procedures.

Anyway we note that cutting force plateau is normally used to check the steady-state conditions in cutting [79] and, in this application, the cutting force is already stabilized when the simulation is stopped.

\section{Concluding remarks}

We have presented a stabilized formulation that allows us to simulate the viscoplastic flow using the same maxent meshfree interpolants for the pressure and the velocity fields. The new technique produces very good results in benchmark applications in metal forming and orthogonal cutting, that are encouraging to extend the method to more accurate models, where friction and thermal aspects should be considered. The extension to three-dimensional applications, that appears to be straightforward, would also be interesting. Another aspect to be taken into account in order to improve the quality of the results would be the implementation of rezoning algorithms. Max-ent approximants, like other meshfree methods, are little influenced by the distribution of the nodes but may loose their accuracy when strong irregularities arise. On the other hand, respect to finite element methods that require time consuming rezoning procedures, this operation takes a negligible computational time in meshfree implementations where the Delaunay triangulation is used only for the integration.

Acknowledgments Francesco Greco acknowledges the travel research fellowship awarded by the Fondo Sociale Europeo. Marino Arroyo and Christian Peco acknowledge the support of the European Research Council under the European Community's 7th Framework Programme (FP7/2007-2013)/ERC grant agreement $\mathrm{nr}$ 240487, and of the Ministerio de Ciencia e Innovacion (DPI201126589). MA acknowledges the support received through the prize "ICREA Academia" for excellence in research, funded by the Generalitat de Catalunya. CP acknowledges FPI-UPC Grant and FPU Ph.D. Grant (Ministry of Science and Innovation, Spain).

\section{References}

1. Kobayashi S, Oh Si, Altan T (1989) Metal forming and the finiteelement method. Oxford University Press

2. Peric D, Owen DRJ (2004) Computational modeling of forming processes. Wiley

3. Babuska I, Aziz AK (1976) On the angle condition in the finite element method. SIAM J Numer Anal 13(2):214-226
4. Zienkiewicz OC, Godbole PN (1974) Flow of plastic and viscoplastic solids with special reference to extrusion and forming processes. Int J Numer Methods Eng 8(1):1-16

5. Zienkiewicz OC (1984) Flow formulation for numerical solution of forming processes. Wiley, Chichester

6. Hwu YJ, Lenard JG (1988) A finite element study of flat rolling. J Eng Mater Technol 110(1):22-27

7. Belytschko T, Kennedy JM (1978) Computer models for subassembly simulation. Nucl Eng Des 49:17-38

8. Liu WK, Herman C, Jiun-Shyan C, Ted B (1988) Arbitrary lagrangian-eulerian petrov-galerkin finite elements for nonlinear continua. Comput Methods Appl Mech Eng 68(3):259-310

9. Yu-Kan H, Liu WK (1993) An ale hydrodynamic lubrication finite element method with application to strip rolling. Int $\mathrm{J}$ Numer Methods Eng 36(5):855-880

10. Belytschko T, Krongauz Y, Organ D, Fleming M, Meshless PK (1996) An overview and recent developments. Comput Methods Appl Mech Eng 139:3-47

11. Puso MA, Solberg J (2006) A stabilized nodally integrated tetrahedral. Int J Numer Methods Eng 67(6):841-867

12. Quak W, Boogaard AH, González D, Cueto E (2011) A comparative study on the performance of meshless approximations and their integration. Comput Mech 48(2):121-137

13. Krysl P, Kagey H (2012) Reformulation of nodally integrated continuum elements to attain insensitivity to distortion. Int $\mathrm{J}$ Numer Methods Eng 90(7):805-818

14. Greco F, Filice L, Alfaro I, Cueto E (2011) On the performances of different nodal integration techniques and their stabilization. In: Proceedings Computational Plast XI - Fundamentals and Application Conference, pp 1455-1466

15. Greco F, Umbrello D, Di Renzo S, Filice L, Alfaro I, Cueto E (2011) Application of the nodal integrated finite element method to cutting, A preliminary comparison with the traditional fem approach. Adv Mater Res 223:172-181

16. Quak W (October 2011) On meshless and nodal-based numerical methods for forming processes, $\mathrm{PhD}$ thesis. Enschede, The Netherlands

17. Belytschko T, Lu YY, Gu L (1994) Element-free galerkin methods. Int J Numer Methods Eng 37(2):229-256

18. Liu WK, Jun S, Yi FZ (1995) Reproducing kernel particle methods. Int J Numer Methods Fluids 20(8-9):1081-1106

19. Huerta A, Fernández-Méndez S (2000) Enrichment and coupling of the finite element and meshless methods. Int J Numer Methods Eng 48:1615-1636

20. Sukumar N, Moran B, Belytschko T (1998) The natural element method in solid mechanics. Int J Numer Methods Eng 43(5):839887

21. Preparata FP, Shamos MI (1985) Computational geometry: an introduction. Springer, New York

22. Cueto E, Doblaré M, Gracia L (2000) Imposing essential boundary conditions in the natural element method by means of densityscaled ?-shapes. Int J Numer Methods Eng 49(4):519-546

23. Alfaro I, Yvonnet J, Chinesta F, Cueto E (2007) A study on the performance of natural neighbour-based galerkin methods. Int $\mathbf{J}$ Numer Methods Eng 71(12):1436-1465

24. Sukumar N (2004) Construction of polygonal interpolants: a maximum entropy approach. Int J Numer Methods Eng 61(12):21592181

25. Arroyo M, Ortiz M (2006) Local maximum-entropy approximation schemes: a seamless bridge between finite elements and meshfree methods. Int J Numer Methods Eng 65(13):2167-2202

26. Rosolen A, Millán D, Arroyo M (2010) On the optimum support size in meshfree methods: a variational adaptivity approach with maximum entropy approximants. Int J Numer Methods Eng 82(7):868-895 
27. Rosolen A, Arroyo M (2013) Blending isogeometric analysis and local maximum entropy meshfree approximants. Comput Methods Appl Mech Eng 264:95-107

28. Millán D, Rosolen A, Arroyo M (2011) Thin shell analysis from scattered points with maximum-entropy approximants. Int $\mathbf{J}$ Numer Methods Eng 85(6):723-751

29. Millán D, Rosolen A, Arroyo M (2013) Nonlinear manifold learning for meshfree finite deformation thin shell analysis. Int J Numer Methods Eng 93:685-713

30. Millán D, Arroyo M (2013) Nonlinear manifold learning for model reduction in finite elastodynamics. Comput Methods Appl Mech Eng 261262(0):118-131

31. Millán D, Arroyo M, Hashemian B (2013) B. Biasing molecular dynamics simulations with smooth and nonlinear data-driven collective variables. Submitted to Journal of Chemical Physics

32. Arroyo PAI, M Abdollahi A (2013) Effect of flexoelectricity on the electromechanical response of nano cantilever beams. Submitted to Journal of Computational Physics

33. Rosolen A, Peco C, Arroyo M (2013) An adaptive meshfree method for phase-field models of biomembranes Part I: approximation with maximum-entropy basis functions. J Comput Phys 249(0):303-319

34. Peco C, Rosolen A, Arroyo M (2013) An adaptive meshfree method for phase-field models of biomembranes Part II: a lagrangian approach for membranes in viscous fluids. Chin $\mathbf{J}$ Comput Phys 249(0):320-336

35. Rabczuk MD, Arroyo TM, Amiri F (2013) Phase-field modeling of fracture mechanics in linear thin shells. J Appl Math

36. Quaranta G, Kunnath SK, Sukumar N (2012) Maximum-entropy meshfree method for nonlinear static analysis of planar reinforced concrete structures. Eng Struct 42:179-189

37. Cyron CJ, Nissen K, Gravemeier V, Wall WA (2010) Stable meshfree methods in fluid mechanics based on greens functions. Comput Mech 46(2):287-300

38. Bonet J, Kulasegaram S (2000) Correction and stabilization of smooth particle hydrodynamics methods with applications in metal forming simulations. Int J Numer Methods Eng 47(6):11891214

39. Guo YM, Nakanishi K (2003) A backward extrusion analysis by the rigidplastic integrallessmeshless method. J Mater Process Technol 13(140):19-24. Proceedings of the 6th Asia Pacific Conference on materials Processing

40. Wen H, Dong X, Yan C, Ruan X (2007) Three dimension profile extrusion simulation using meshfree method. Int J Adv Manuf Tech 34(3-4):270-276

41. Wu CT, Chen JS, Pan C, Roque C (1998) A lagrangian reproducing kernel particle method for metal forming analysis. Comput Mech, 289

42. Chen JS, Roque CMOL, Chunhui P, Button ST (1998) Analysis of metal forming process based on meshless method. J Mater Process Technol, 642-646

43. Yoon S, Chen JS (2002) Accelerated meshfree method for metal forming simulation. Finite Elem Anal Des 38(10):937-948

44. Alfaro I, Yvonnet J, Cueto E, Chinesta F, Doblaré M (2006) Meshless methods with application to metal forming. Comput Methods Appl Mech Eng 195(489):6661-6675

45. Alfaro I, González D, Bel D, Cueto E, Doblar M, Chinesta F (2006) advances in the meshless simulation of aluminium extrusion and other related forming processes. Archives Comput Methods Eng 13(1):3-43

46. Alfaro I, Bel D, Cueto E, Doblaré M, Chinesta F (2006) Threedimensional simulation of aluminium extrusion by the -shape based natural element method. Comput Methods Appl Mech Eng 195(33-36):4269-4286
47. Alfaro I, Gagliardi F, Olivera J, Cueto E, Filice L, Chinesta F (2009) Simulation of the extrusion of hollow profiles by natural element methods. Int J Mater Form 2(Supplement 1):597-600

48. Cueto E, Chinesta F (2013) Meshless methods for the simulation of material forming. Int J Mater Form, 1-19

49. Quak W, Gonzalez D, Cueto E, van den Boogaard AH (2009) On the use of local max-ent shape functions for the simulation of forming processes. In: Onate E, Owen DRJ (eds) X International Conference on Computational Plasticity, COMPLAS X. CIMNE, Barcelona, Spain

50. Chenot JL, Bellet M (1992) Numerical Modelling of Material Deformation Processes. In: Hartley P, Pillinger I, Sturgess C (eds). Springer, London, pp 179-224

51. Dolbow J, Belytschko T (1999) Volumetric locking in the element free Galerkin method. Int J Numer Methods Eng 46(6):925-942

52. González D, Cueto E, Doblaré M (2004) Volumetric locking in natural neighbour Galerkin methods. Int J Numer Methods Eng 61(4):611-632

53. Brezzi F (1974) On the existence, uniqueness and approximation of saddle-point problems arising from lagrangian multipliers. ESAIM: Math Model Numer Anal Modél Math Anal Numér 8(R2):129-151

54. Babuka I (1973) The finite element method with lagrangian multipliers. Numerische Mathematik 20(3):179-192

55. Li J, He Y, Chen Z (2009) Performance of several stabilized finite element methods for the stokes equations based on the lowest equal-order pairs. Computing 86(1):37-51

56. Ortiz A, Puso MA, Sukumar N (2010) Maximum-entropy meshfree method for compressible and near-incompressible elasticity. Comput Methods Appl Mech Eng 199(25-28):1859-1871

57. Arnold DN, Brezzi F, Fortin M (1984) A stable finite element for the stokes equations. CALCOLO 21(4):337-344

58. Ortiz A, Puso MA, Sukumar N (2011) Maximum-entropy meshfree method for incompressible media problems. Finite Elem Anal Des 47(6):572-585

59. Cyron CJ, Arroyo M, Ortiz M (2009) Smooth, second order, nonnegative meshfree approximants selected by maximum entropy. Int J Numer Methods Eng 79(13):1605-1632

60. Rosolen A, Millán D, Arroyo M (2012) Second order convex maximum entropy approximants with applications to high order PDE. Int J Numer Methods Eng

61. González D, Cueto E, Doblaré M (2010) A higher-order method based on local maximum entropy approximation. Int J Numer Methods Eng 83(6):741-764

62. Bompadre A, Perotti LE, Cyron C, Ortiz M (2012) Convergent meshfree approximation schemes of arbitrary order and smoothness. Comput Methods Appl Mech Eng 221-222:83-103

63. Puso MA, Chen JS, Zywicz E, Elmer W (2008) Meshfree and finite element nodal integration methods. Int J Numer Methods Eng 74(3):416-446

64. Codina R (1998) Comparison of some finite element methods for solving the diffusion-convection-reaction equation. Comput Methods Appl Mech Eng 156(14s):185-210

65. Barth T, Bochev P, Gunzburger M, Shadid J (2004) A taxonomy of consistently stabilized finite element methods for the stokes problem. SIAM J Sci Comput 25(5):1585-1607

66. Bochev P, Gunzburger M (2004) An absolutely stable pressurepoisson stabilized finite element method for the stokes equations. SIAM J Numer Anal 42(3):1189-1207

67. Peco C, Rosolen A, Arroyo M (2013) Estabilización de las ecuaciones de stokes con aproximantes locales de máxima entropía. Submitted to RIMNI

68. Brezzi F, Pitkaranta J (1984) On the stabilization of finite element approximations of the Stokes equations. Notes on Numerical 
Fluid Mechanics, Efficient Solutions of Elliptic Systems, vol 10. Viewig, Braunschweig, pp 11-19

69. Greco F, Sukumar N (2013) Derivatives of maximum-entropy basis functions on the boundary: theory and computations. Int $\mathbf{J}$ Numer Methods Eng 94(12):1123-1149

70. Hughes TJR, Franca LP, Balestra M (1986) A new finite element formulation for computational fluid dynamics: V. circumventing the babuka-brezzi condition: a stable Petrov-Galerkin formulation of the stokes problem accommodating equalorder interpolations. Comput Methods Appl Mech Eng 59(1): 85-99

71. Jansen KE, Collis SS, Whiting C, Shakib F (1999) A better consistency for low-order stabilized finite element methods. Comput Methods Appl Mech Eng 174(1-2):153-170

72. Harari I, Hughes TJR (1992) What are C and h?: Inequalities for the analysis and design of finite element methods. Comput Methods Appl Mech Eng 97(2):157-192
73. Wriggers P (2003) Computational contact mechanics. Comput Mech 32:141-141

74. Hormann K, Sukumar N (2008) Maximum entropy coordinates for arbitrary polytopes. In: Proceedings of SGP 2008

75. Edelsbrunner H, Kirkpatrick D, Seidel R (1983) On the shape of a set of points in the plane. IEEE Trans Inform Theory 29(4):551559

76. Kalpakjian S (1992) Manufacturing Processes for Engineering Materials, 5/e (New Edition). Pearson Education

77. Quak W., Boogaard A. H., Hutink J (2009) Meshless methods and forming processes. Int J Mater Form 2(1):585-588

78. Ceretti E, Taupin E, Altan T (1997) Simulation of metal flow and fracture applications in orthogonal cutting, blanking, and cold extrusion. CIRP Ann-Manuf Technol 46(1):187-190

79. Arrazola PJ, zel T, Umbrello D, Davies M, Jawahir IS (2013) Recent advances in modelling of metal machining processes. CIRP Ann-Manuf Technol 62(2):695-718 